\title{
Solutions of Several Coupled Discrete Models in terms of Lamé Polynomials of Arbitrary Order
}

\author{
Avinash Khare \\ Raja Ramanna Fellow, Indian Institute of Science Education and Research, Pune 411021, India \\ Avadh Saxena \\ Theoretical Division and Center for Nonlinear Studies, Los Alamos National Laboratory, Los Alamos, \\ NM 87545, USA \\ Apoorva Khare \\ Departments of Mathematics and Statistics, Stanford University, CA 94305, USA
}

\begin{abstract}
:
Coupled discrete models abound in several areas of physics. Here we provide an extensive set of exact quasiperiodic solutions of a number of coupled discrete models in terms of Lamé polynomials of arbitrary order. The models discussed are (i) coupled Salerno model, (ii) coupled Ablowitz-Ladik model, (iii) coupled $\phi^{4}$ model, and (iv) coupled $\phi^{6}$ model. In all these cases we show that the coefficients of the Lamé polynomials are such that the Lamé polynomials can be reexpressed in terms of Chebyshev polynomials of the relevant Jacobi elliptic function.
\end{abstract}




\section{Introduction}

In a recent paper [1] we have obtained solutions of a number of coupled discrete models in terms of Lamé polynomials of order one and two. The purpose of the present paper is to show that in the same models one can in fact obtain solutions in terms of Lamé polynomials (and hence trigonometric and hyperbolic polynomials) of arbitrary order. In particular, we obtain solutions of (i) Coupled Salerno model (ii) coupled Ablowitz-Ladik (AL) model (iii) coupled $\phi^{6}$ model (iv) coupled $\phi^{4}$ model, in terms of Lamé (and hence trigonometric and hyperbolic) polynomials of arbitrary order. As an illustration, we confine our discussion to coupled Salerno model and then show how similar solutions also exist in coupled Ablowitz-Ladik, coupled $\phi^{4}$ and coupled $\phi^{6}$ models. Quite remarkably, we find that the coefficients of the Lamé polynomials are such that the Lame polynomials can be reexpressed as Chebyshev polynomials of the relevant Jacobi elliptic function.

There are many physical situations where a discrete field theory is appropriate to model the phenomena of interest with a specific coupling between the two fields. This fact serves as one of the main motivation for the results presented here. A technologically important phenomenon of current intense interest is the coexistence of magnetism and ferroelectricity (i.e., magnetoelectricity) in a given material. This is a highly desired functionality in applications involving cross-field response such as electric field control of magnetism and magnetic field control of electric polarization, switching and actuation. More generally, presence of two or more such ferroic properties (e.g. polarization, magnetization, strain) is referred to as multiferroic behavior [4. In recent years, two different classes of (single phase) multiferroics, namely the orthorhombically distorted perovskites [5] and rare earth hexagonal structures [6], have emerged in addition to other related structures. The hexagonal materials show axial polarization and magnetic domain walls in the basal planes. The latter can be modeled by a coupled $\phi^{4}$ model [7] in the presence of a magnetic field. Coupled $\phi^{4}$ models [8, 9, 10] are useful in the study of many ferroelectric and other second order phase transitions. For multiferroic materials the relevant coupled $\phi^{4}$ model [7] has a biquadratic coupling. In contrast, the coupled $\phi^{4}$ model for a surface phase transition with hydration forces [10], which arises in the biophysics context, has a bilinear coupling. Other types of couplings are also known for structural phase transitions involving strain tensor components [11]. 
There are many examples of coupled discrete Ablowitz-Ladik, coupled discrete Salerno and coupled saturated discrete nonlinear Schrödinger (DNLS) models known in the literature [12, 13, 14]. In addition, there are analogous coupled models known in the field theoretic contexts [15, 16]. Several related models have been discussed in the literature and their soliton solutions have been obtained [17, 18, 19, 20, 21, 22, 23, 24] including periodic ones [25, 26, 27].

The paper is organized as follows. In Section II we provide the solutions for the coupled Salerno model in terms of Lamé polynomials of order three and four. Based on these results as well those obtained in [1], in Section III we generalize these results and conjecture solutions in terms of Lamé (and hence trigonometric and hyperbolic) polynomials of arbitrary order. In Section IV we show that these Lamé polynomials can be reexpressed as Chebyshev polynomials of the relevant Jacobi elliptic function. This also proves that our proposed Lamé polynomials of arbitrary order are indeed solutions of the coupled equations. In Section V we show how the coupled Ablowitz-Ladik, coupled discrete $\phi^{6}$, and coupled $\phi^{4}$ models also admit solutions in terms of Lamé polynomials of arbitrary order. Section VI contains the summary of main results and possible future directions.

\section{Coupled Salerno Model}

As discussed in a recent paper [1, the field equations of the coupled Salerno model are given by

$$
\begin{gathered}
i d u_{n} / d t+\left[u_{n+1}+u_{n-1}-2 u_{n}\right]+\left(\mu_{1}\left|u_{n}\right|^{2}+\mu_{2}\left|v_{n}\right|^{2}\right)\left[u_{n+1}+u_{n-1}+\frac{\nu_{1}-2 \mu_{1}}{\mu_{1}} u_{n}\right]=0 \\
i d v_{n} / d t+\left[v_{n+1}+v_{n-1}-\left(2+\frac{\nu_{1} \mu_{2}}{\mu_{1}^{2}}-\frac{\nu_{2}}{\mu_{2}}\right) v_{n}\right]+\left(\mu_{1}\left|u_{n}\right|^{2}+\mu_{2}\left|v_{n}\right|^{2}\right)\left[v_{n+1}+v_{n-1}+\frac{\nu_{2}-2 \mu_{2}}{\mu_{2}} v_{n}\right]=0 .
\end{gathered}
$$

In ([1]) we have already obtained solutions of these coupled equations in terms of Lamé polynomials of order one and two. We now show that the same model also admits Lamé polynomial solutions of arbitrary order.

As in [1], we start with the ansatz

$$
u_{n}=f_{n} \exp \left[-i\left(\omega_{1} t+\delta_{1}\right)\right], \quad v_{n}=g_{n} \exp \left[-i\left(\omega_{2} t+\delta_{2}\right)\right]
$$


then it is easily shown that the above coupled equations take the form

$$
\begin{gathered}
\left(\omega_{1}-2\right) f_{n}+\left(f_{n+1}+f_{n-1}\right)\left(\mu_{1} f_{n}^{2}+\mu_{2} g_{n}^{2}+1\right)+\left(\mu_{1} f_{n}^{2}+\mu_{2} g_{n}^{2}\right) \frac{\left(\nu_{1}-2 \mu_{1}\right)}{\mu_{1}} f_{n}=0, \\
\left(\omega_{2}-2+\frac{\nu_{1} \mu_{2}}{\mu_{1}^{2}}-\frac{\nu_{2}}{\mu_{2}}\right) g_{n}+\left(\mu_{1} f_{n}^{2}+\mu_{2} g_{n}^{2}+1\right)\left(g_{n+1}+g_{n-1}\right)+\left(\mu_{1} f_{n}^{2}+\mu_{2} g_{n}^{2}\right) \frac{\left(\nu_{2}-2 \mu_{2}\right)}{\mu_{2}} g_{n}=0 .
\end{gathered}
$$

It is clear from Eqs. (4) and (5) that in general these coupled equations will have exact solutions if

$$
1+\mu_{1} f_{n}^{2}+\mu_{2} g_{n}^{2}=0
$$

and in that case

$$
\omega_{1}=\frac{\nu_{1}}{\mu_{1}}, \quad \omega_{2}=\frac{\nu_{1} \mu_{2}}{\mu_{1}^{2}} .
$$

We now show that Eq. (6) has solutions in terms of Lamé polynomials of arbitrary order. In particular, we show that at every order there are three distinct solutions satisfying Eq. (6). As a first step, let us explicitly obtain solutions of Eq. (6) in terms of Lamé polynomials of order three and four. (It may be noted that we have already obtained such solutions before in terms of Lame polynomials of order one and two [1].)

\subsection{Lamé Polynomial Solutions of Order Three}

It is easily checked that one of the exact solution to Eq. (6) is

$$
f_{n}=\operatorname{dn}\left[\beta\left(n+c_{2}\right), m\right]\left(A \operatorname{dn}^{2}\left[\beta\left(n+c_{2}\right), m\right]+B\right), \quad g_{n}=\sqrt{m} \operatorname{sn}\left[\beta\left(n+c_{2}\right), m\right]\left(C \operatorname{dn}^{2}\left[\beta\left(n+c_{2}\right), m\right]+D\right),
$$

provided

$$
\begin{aligned}
& \mu_{1}, \mu_{2}<0, \quad\left|\mu_{1}\right| A^{2}=\left|\mu_{2}\right| C^{2}, \quad\left|\mu_{2}\right| D^{2}=1 \\
& \left|\mu_{1}\right|(A+B)^{2}=1, \quad\left|\mu_{1}\right|\left(A^{2}+2 A B\right)=2 C D\left|\mu_{2}\right|
\end{aligned}
$$

Here $c_{2}$ is an arbitrary constant signifying discrete translation invariance. Also notice that for this solution, the width $\beta$ is also completely arbitrary. We shall see that all the solutions discussed in this paper are valid for arbitrary $c_{2}$ and width $\beta$. On solving, we find that

$$
\sqrt{\left|\mu_{1}\right|} A=4, B=-\frac{3}{4} A, \quad \sqrt{\left|\mu_{2}\right|} C=4, \quad C=-4 D .
$$


Since the field Eqs. (4) and (5) are invariant under $f_{n} \rightarrow \pm f_{n}, g_{n} \rightarrow \pm g_{n}$, one can trivially write three other solutions from here. Note that $\beta$ is completely arbitrary. Using the fact that $\operatorname{dn}(x, m)$ has period $2 K(m)$ while $\operatorname{cn}(x, m)$ and $\operatorname{sn}(x, m)$ are periodic functions with period $4 K(m)$, it follows that for the solution (8), $f_{n}, g_{n}$ and hence $u_{n}, v_{n}$ satisfy the boundary condition

$$
u_{n+\frac{2 K(m)}{\beta}}=u_{n}, \quad v_{n+\frac{4 K(m)}{\beta}}=v_{n} .
$$

Here $K(m)$ is the complete elliptic integral of the first kind.

Another solution to Eq. (6) is

$$
f_{n}=\operatorname{cn}\left[\beta\left(n+c_{2}\right), m\right]\left(A \operatorname{cn}^{2}\left[\beta\left(n+c_{2}\right), m\right]+B\right), \quad g_{n}=\operatorname{sn}\left[\beta\left(n+c_{2}\right), m\right]\left(C \operatorname{cn}^{2}\left[\beta\left(n+c_{2}\right), m\right]+D\right),
$$

provided $A, B, C, D$ are again given by Eq. (10). Note however that the solution (12) is distinct from the solution (8). In particular, while for the solution (8), $u_{n}, v_{n}$ satisfy the boundary condition (11), for the solution (12), $f_{n}, g_{n}$ and hence $u_{n}, v_{n}$ satisfy the boundary condition

$$
u_{n+\frac{2 K(m)}{\beta}}=u_{n}, \quad v_{n+\frac{2 K(m)}{\beta}}=v_{n} .
$$

However, in the limit $m=1$, both the solutions (8) and (12) go over to the hyperbolic solution

$$
f_{n}=A \operatorname{sech}^{3}\left[\beta\left(n+c_{2}\right)\right]+B \operatorname{sech}\left[\beta\left(n+c_{2}\right)\right], \quad g_{n}=\tanh \left[\beta\left(n+c_{2}\right)\right]\left(C \operatorname{sech}^{2}\left[\beta\left(n+c_{2}\right)\right]+D\right)
$$

Further, in the limit $m=0$, the solution (12) goes over to the trigonometric solution

$$
f_{n}=\cos \left[\beta\left(n+c_{2}\right)\right]\left(A \cos ^{2}\left[\beta\left(n+c_{2}\right)\right]+B\right), \quad g_{n}=\sin \left[\beta\left(n+c_{2}\right)\right]\left(C \cos ^{2}\left[\beta\left(n+c_{2}\right)\right]+D\right)
$$

All the four solutions discussed above are valid only if $\mu_{1}, \mu_{2}<0$. If instead $\mu_{1}, \mu_{2}$ have opposite signs then also one has solutions to Eq. (6). One such solution is

$$
f_{n}=\frac{1}{\operatorname{dn}\left[\beta\left(n+c_{2}\right), m\right]}\left(\frac{A}{\operatorname{dn}^{2}\left[\beta\left(n+c_{2}\right), m\right]}+B\right), \quad g_{n}=\frac{\sqrt{m} \operatorname{sn}\left[\beta\left(n+c_{2}\right), m\right]}{\operatorname{dn}\left[\beta\left(n+c_{2}\right), m\right]}\left(\frac{C}{\operatorname{dn}^{2}\left[\beta\left(n+c_{2}\right), m\right]}+D\right),
$$

provided $\mu_{1}<0, \mu_{2}>0$ while $A, B, C, D$ are still given by Eq. (10). Note that for this solution $f_{n}, g_{n}$ and hence $u_{n}, v_{n}$ satisfy the boundary condition (11). Further, if we interchange $f_{n}$ and $g_{n}$, then $\mu_{1}>0, \mu_{2}<0$. In the limit $m=1$, this solution goes over to the hyperbolic solution

$$
f_{n}=A \cosh ^{3}\left[\beta\left(n+c_{2}\right)\right]+B \cosh \left[\beta\left(n+c_{2}\right)\right], \quad g_{n}=\sinh \left[\beta\left(n+c_{2}\right)\right]\left(C \cosh ^{2}\left[\beta\left(n+c_{2}\right)\right]+D\right)
$$




\subsection{Lamé Polynomial Solutions of Order Four}

It is easily checked that one of the exact solution to Eq. (6) is

$$
\begin{aligned}
& f_{n}=A \operatorname{dn}^{4}\left[\beta\left(n+c_{2}\right), m\right]+B \operatorname{dn}^{2}\left[\beta\left(n+c_{2}\right), m\right]+C, \\
& g_{n}=\sqrt{m} \operatorname{sn}\left[\beta\left(n+c_{2}\right), m\right] \operatorname{dn}\left[\beta\left(n+c_{2}\right), m\right]\left(D \operatorname{dn}^{2}\left[\beta\left(n+c_{2}\right), m\right]+E\right),
\end{aligned}
$$

provided

$$
\begin{aligned}
& \mu_{1}, \mu_{2}<0, \quad\left|\mu_{1}\right| A^{2}=\left|\mu_{2}\right| D^{2}, \quad\left|\mu_{1}\right| C^{2}=1, \quad A(A+2 B)\left|\mu_{1}\right|=2 D E\left|\mu_{2}\right|, \\
& \left|\mu_{1}\right|(A+B)^{2}+2 A C\left|\mu_{1}\right|=\left|\mu_{2}\right| E^{2}, \quad 2 B C\left|\mu_{1}\right|=-\left|\mu_{2}\right| E^{2} .
\end{aligned}
$$

On solving, we find that

$$
\sqrt{\left|\mu_{1}\right|} C=1, A=-B=8 C, \quad \sqrt{\left|\mu_{2}\right|} E=-4, \quad D=-2 E .
$$

For the solution (18), $f_{n}, g_{n}$ and hence $u_{n}, v_{n}$ satisfy the boundary condition (11).

Another solution to Eq. (6) is

$$
\begin{aligned}
& f_{n}=A \operatorname{cn}^{4}\left[\beta\left(n+c_{2}\right), m\right]+B \operatorname{cn}^{2}\left[\beta\left(n+c_{2}\right), m\right]+C, \\
& g_{n}=\operatorname{sn}\left[\beta\left(n+c_{2}\right), m\right] \operatorname{cn}\left[\beta\left(n+c_{2}\right), m\right]\left(D \operatorname{cn}^{2}\left[\beta\left(n+c_{2}\right), m\right]+E\right),
\end{aligned}
$$

provided $A, B, C, D, E$ are again given by Eq. (20). Note however that the solution (21) is distinct from the solution (18). In particular, while for the solution (18), $u_{n}, v_{n}$ satisfy the boundary condition (11), for the solution (21), $f_{n}, g_{n}$ and hence $u_{n}, v_{n}$ satisfy the boundary condition (13).

However, in the limit $m=1$, both the solutions (18) and (21) go over to the hyperbolic solution

$$
\begin{aligned}
& f_{n}=A \operatorname{sech}^{4}\left[\beta\left(n+c_{2}\right)\right]+B \operatorname{sech}^{2}\left[\beta\left(n+c_{2}\right)\right]+C, \\
& g_{n}=\tanh \left[\beta\left(n+c_{2}\right)\right] \operatorname{sech}\left[\beta\left(n+c_{2}\right)\right]\left(D \operatorname{sech}^{2}\left[\beta\left(n+c_{2}\right)\right]+E\right),
\end{aligned}
$$

Further, in the limit $m=0$, the solution (21) goes over to the trigonometric solution

$$
\begin{aligned}
& f_{n}=A \cos ^{4}\left[\beta\left(n+c_{2}\right)\right]+B \cos ^{2}\left[\beta\left(n+c_{2}\right)\right]+C, \\
& g_{n}=\sin \left[\beta\left(n+c_{2}\right)\right] \cos \left[\beta\left(n+c_{2}\right)\right]\left(D \cos ^{2}\left[\beta\left(n+c_{2}\right)\right]+E\right),
\end{aligned}
$$


All the four solutions discussed above are valid only if $\mu_{1}, \mu_{2}<0$. If instead $\mu_{1}, \mu_{2}$ have opposite signs then also one has solutions to Eq. (66). One such solution is

$$
\begin{aligned}
& f_{n}=\frac{A}{\operatorname{dn}^{4}\left[\beta\left(n+c_{2}\right), m\right]}+\frac{B}{\operatorname{dn}^{2}\left[\beta\left(n+c_{2}\right), m\right]}+C, \\
& g_{n}=\sqrt{m} \operatorname{sn}\left[\beta\left(n+c_{2}\right), m\right]\left(\frac{D}{\operatorname{dn}^{4}\left[\beta\left(n+c_{2}\right), m\right]}+\frac{E}{\operatorname{dn}^{2}\left[\beta\left(n+c_{2}\right), m\right]}\right),
\end{aligned}
$$

provided $\mu_{1}<0, \mu_{2}>0$ while $A, B, C, D, E$ are still given by Eq. (20). For this solution, $f_{n}, g_{n}$ and hence $u_{n}, v_{n}$ satisfy the boundary condition (11). Note that if we interchange $f_{n}$ and $g_{n}$, then $\mu_{1}>0, \mu_{2}<0$. In the limit $m=1$, this solution goes over to the hyperbolic solution

$$
\begin{aligned}
& f_{n}=A \cosh ^{4}\left[\beta\left(n+c_{2}\right)\right]+B \cosh ^{2}\left[\beta\left(n+c_{2}\right)\right]+C, \\
& g_{n}=\sinh \left[\beta\left(n+c_{2}\right)\right]\left(D \cosh ^{3}\left[\beta\left(n+c_{2}\right)\right]+E \cosh \left[\beta\left(n+c_{2}\right)\right]\right) .
\end{aligned}
$$

\section{General Results}

Looking at the structure of the solutions in terms of Lamé polynomials of order one to four, it is easy to generalize and write down the solutions of Eq. (6) in terms of Lamé polynomials of arbitrary order. For this we need to divide the discussion into two parts depending on if we are considering Lamé polynomials of odd or even order.

\section{Case I: Lame Polynomials of odd order}

One of the solutions can be written in the form (note $n$ is an odd integer)

$$
\begin{aligned}
f_{n} & =\sum_{k=1}^{(n+1) / 2} A_{k}\left(\operatorname{dn}\left[\beta\left(n+c_{2}\right), m\right]\right)^{2 k-1}, \\
g_{n} & =\sqrt{m} \operatorname{sn}\left[\beta\left(n+c_{2}\right), m\right] \sum_{k=1}^{(n+1) / 2} B_{k}\left(\operatorname{dn}\left[\beta\left(n+c_{2}\right), m\right]\right)^{2 k-2} .
\end{aligned}
$$

For this solution, $f_{n}, g_{n}$ and hence $u_{n}, v_{n}$ satisfy the boundary condition (11). Note that there are $(n+1) / 2$ number of terms in both $f_{n}$ and $g_{n}$. 
Another solution is given by

$$
\begin{aligned}
& f_{n}=\sum_{k=1}^{(n+1) / 2} A_{k}\left(\operatorname{cn}\left[\beta\left(n+c_{2}\right), m\right]\right)^{2 k-1}, \\
& g_{n}=\operatorname{sn}\left[\beta\left(n+c_{2}\right), m\right] \sum_{k=1}^{(n+1) / 2} B_{k}\left(\operatorname{cn}\left[\beta\left(n+c_{2}\right), m\right]\right)^{2 k-2} .
\end{aligned}
$$

For this solution, $f_{n}, g_{n}$ and hence $u_{n}, v_{n}$ satisfy the boundary condition (13).

In the limit $m=1$, both the solutions (26) and (27) go over to the hyperbolic solution

$$
\begin{aligned}
f_{n} & =\sum_{k=1}^{(n+1) / 2} A_{k}\left(\operatorname{sech}\left[\beta\left(n+c_{2}\right)\right]\right)^{2 k-1}, \\
g_{n} & =\tanh \left[\beta\left(n+c_{2}\right)\right] \sum_{k=1}^{(n+1) / 2} B_{k}\left(\operatorname{sech}\left[\beta\left(n+c_{2}\right)\right]\right)^{2 k-2} .
\end{aligned}
$$

Further, in the limit $m=0$, the solution (27) goes over to the trigonometric solution

$$
\begin{aligned}
f_{n} & =\sum_{k=1}^{(n+1) / 2} A_{k}\left(\cos \left[\beta\left(n+c_{2}\right)\right]\right)^{2 k-1}, \\
g_{n} & =\sin \left[\beta\left(n+c_{2}\right)\right] \sum_{k=1}^{(n+1) / 2} B_{k}\left(\cos \left[\beta\left(n+c_{2}\right)\right]\right)^{2 k-2} .
\end{aligned}
$$

Note that these four solutions are valid in case $\mu_{1}, \mu_{2}<0$. However, if $\mu_{1}$ and $\mu_{2}$ have opposite signs, say $\mu_{1}<0, \mu_{2}>0$, then the solution is given by

$$
\begin{aligned}
f_{n} & =\sum_{k=1}^{(n+1) / 2} \frac{A_{k}}{\left(\operatorname{dn}\left[\beta\left(n+c_{2}\right), m\right]\right)^{2 k-1}}, \\
g_{n} & =\sqrt{m} \operatorname{sn}\left[\beta\left(n+c_{2}\right), m\right] \sum_{k=1}^{(n+1) / 2} \frac{B_{k}}{\left(\operatorname{dn}\left[\beta\left(n+c_{2}\right), m\right]\right)^{2 k-1}} .
\end{aligned}
$$

For this solution, $f_{n}, g_{n}$ and hence $u_{n}, v_{n}$ satisfy the boundary condition (11). In the limit $m=1$, this solution goes over to the hyperbolic solution

$$
\begin{aligned}
f_{n} & =\sum_{k=1}^{(n+1) / 2} A_{k}\left(\cosh \left[\beta\left(n+c_{2}\right)\right]\right)^{2 k-1}, \\
g_{n} & =\tanh \left[\beta\left(n+c_{2}\right)\right] \sum_{k=1}^{(n+1) / 2} B_{k}\left(\cosh \left[\beta\left(n+c_{2}\right)\right]\right)^{2 k-1} .
\end{aligned}
$$

On substituting any of the expressions for $f_{n}, g_{n}$ as given by Eqs. (26) to (31) in Eq. (6), we obtain $n+1$ equations which determine the $n+1$ parameters $A_{k}, B_{k}$. While all $A_{k}$ 's are numbers in units of $\frac{1}{\sqrt{\left|\mu_{1}\right|}}$, 
all $B_{k}$ 's are numbers in units of $\frac{1}{\sqrt{\left|\mu_{2}\right|}}$. For simplicity from now onwards we will merely give the numerical values of $A_{k}, B_{k}$ and it is understood that they are in units of $\frac{1}{\sqrt{\left|\mu_{1}\right|}}$ and $\frac{1}{\sqrt{\left|\mu_{2}\right|}}$, respectively. Some of the relations are

$$
\begin{aligned}
& A_{(n+1) / 2}^{2}=B_{(n+1) / 2}^{2}, \\
& 2 A_{(n+1) / 2} A_{(n-1) / 2}+B_{(n+1) / 2}^{2}=2 B_{(n+1) / 2} B_{(n-1) / 2}, \\
& A_{(n-1) / 2}^{2}+2 A_{(n+1) / 2} A_{(n-3) / 2}+2 B_{(n+1) / 2} B_{(n-1) / 2}=B_{(n-1) / 2}^{2}+2 B_{(n+1) / 2} B_{(n-3) / 2}, \\
& B_{(n-1) / 2}^{2}+2 A_{(n+1) / 2} A_{(n-5) / 2}+2 B_{(n+1) / 2} B_{(n-3) / 2}+2 A_{(n-1) / 2} A_{(n-3) / 2} \\
& =2 B_{(n-1) / 2} B_{(n-3) / 2}+2 B_{(n+1) / 2} B_{(n-5) / 2}, \\
& B_{1}^{2}=1, \quad A_{1}^{2}+2 B_{1} B_{2}=B_{1}^{2}, \quad 2 A_{1} A_{2}+B_{2}^{2}+2 B_{1} B_{3}=2 B_{1} B_{2}, \\
& A_{2}^{2}+2 A_{1} A_{3}-B_{2}^{2}+2 B_{2} B_{3}+2 B_{1} B_{4}-2 B_{1} B_{3}=0 .
\end{aligned}
$$

On comparing these results with the exact expressions for $n=1,3$, we conjecture the following general results for arbitrary odd $n$ :

$$
\begin{aligned}
& A_{(n+1) / 2}=B_{(n+1) / 2}=2^{n-1}, \quad A_{(n-1) / 2}=-n 2^{n-3}, \quad B_{(n-1) / 2}=-(n-2) 2^{n-3}, \\
& A_{(n-3) / 2}=\frac{n(n-3)}{2 !} 2^{n-5}, \quad B_{(n-3) / 2}=\frac{(n-3)(n-4)}{2 !} 2^{n-5}, \\
& A_{(n-5) / 2}=-\frac{n(n-4)(n-5)}{3 !} 2^{n-7}, \quad B_{(n-5) / 2}=-\frac{(n-4)(n-5)(n-6)}{3 !} 2^{n-7}, \\
& \sum_{i=1}^{(n+1) / 2} A_{i}=1, \quad \sum_{i=1}^{(n+1) / 2} B_{i}=n .
\end{aligned}
$$

Further, depending on if $n=4 k+3$ or $4 k+1$ we have the following results:

$$
\begin{aligned}
& A_{1}=-n, \quad B_{1}=-1, \quad \text { if } n=4 k+3, \quad k=0,1,2, \ldots \\
& A_{1}=+n, \quad B_{1}=+1, \quad \text { if } n=4 k+1, \quad k=0,1,2, \ldots
\end{aligned}
$$

In fact looking at these general results, we conjecture the following expressions for various $A_{i}, B_{i}$.

$$
\begin{gathered}
A_{\frac{n-(2 k-1)}{2}}=(-1)^{k} \frac{n(n-k-1)(n-k-2) \ldots(n-2 k+1)}{k !} 2^{n-2 k-1}, \quad k=0,2, \ldots, \frac{n-1}{2}, \\
B_{\frac{n-(2 k-1)}{2}}=(-1)^{k} \frac{(n-k-1)(n-k-2) \ldots(n-2 k)}{k !} 2^{n-2 k-1}, \quad k=0,1,2, \ldots, \frac{n-1}{2} .
\end{gathered}
$$


It can be verified that for $n=1,3$, the $A_{i}, B_{i}$ which follow from Eqs. (33) to (35) agree with the values obtained by us above as well as in [1]. Further, for low values of (odd) $n$, it can be numerically checked that the $A_{i}, B_{i}$ which follow from Eqs. (36) and (37) indeed satisfy the various relations which follow by demanding the validity of the constraint relation (6).

In the next section, we will prove that our conjectures hold for all $n$. But first, using Eqs. (33), (36), and (37), we predict that the following identities hold for all odd $n$ :

$$
\begin{gathered}
\sum_{k=0}^{(n-1) / 2}(-1)^{k} \frac{n(n-k-1)(n-k-2) \ldots(n-2 k+1)}{k !} 2^{n-2 k-1}=1 . \\
\sum_{k=0}^{(n-1) / 2}(-1)^{k} \frac{(n-k-1)(n-k-2) \ldots(n-2 k)}{k !} 2^{n-2 k-1}=n .
\end{gathered}
$$

We will see that these identities naturally lead to the proof of our conjectures.

\section{Case II: Lame Polynomials of even order}

In this case there are $n / 2+1$ number of terms in $f_{n}$ and $n / 2$ number of terms in $g_{n}$. In this case, one of the solutions can be written in the form (note $n$ is an even integer)

$$
\begin{aligned}
f_{n} & =\sum_{k=1}^{(n / 2+1)} A_{k}\left(\operatorname{dn}\left[\beta\left(n+c_{2}\right), m\right]\right)^{2(k-1)}, \\
g_{n} & =\sqrt{m} \operatorname{sn}\left[\beta\left(n+c_{2}\right), m\right] \operatorname{dn}\left[\beta\left(n+c_{2}\right), m\right] \sum_{k=1}^{(n / 2)} B_{k}\left(\operatorname{dn}\left[\beta\left(n+c_{2}\right), m\right]\right)^{2(k-1)} .
\end{aligned}
$$

For this solution, $f_{n}, g_{n}$ and hence $u_{n}, v_{n}$ satisfy the boundary condition (11).

Another solution is given by

$$
\begin{aligned}
f_{n} & =\sum_{k=1}^{n / 2+1} A_{k}\left(\operatorname{cn}\left[\beta\left(n+c_{2}\right), m\right]\right)^{2(k-1)}, \\
g_{n} & =\operatorname{sn}\left[\beta\left(n+c_{2}\right), m\right] \operatorname{cn}\left[\beta\left(n+c_{2}\right), m\right] \sum_{k=1}^{n / 2} B_{k}\left(\operatorname{cn}\left[\beta\left(n+c_{2}\right), m\right]\right)^{2(k-1)} .
\end{aligned}
$$

For this solution, $f_{n}, g_{n}$ and hence $u_{n}, v_{n}$ satisfy the boundary condition (13).

In the limit $m=1$, both the solutions (40) and (41) go over to the hyperbolic solution

$$
\begin{aligned}
& f_{n}=\sum_{k=1}^{n / 2+1} A_{k}\left(\operatorname{sech}\left[\beta\left(n+c_{2}\right)\right]\right)^{2(k-1)}, \\
& g_{n}=\tanh \left[\beta\left(n+c_{2}\right)\right] \operatorname{sech}\left[\beta\left(n+c_{2}\right)\right] \sum_{k=1}^{n / 2} B_{k}\left(\operatorname{sech}\left[\beta\left(n+c_{2}\right)\right]\right)^{2(k-1)} .
\end{aligned}
$$


Further, in the limit $m=0$, the solution (41) goes over to the trigonometric solution

$$
\begin{aligned}
& f_{n}=\sum_{k=1}^{n / 2+1} A_{k}\left(\cos \left[\beta\left(n+c_{2}\right)\right]\right)^{2(k-1)}, \\
& g_{n}=\sin \left[\beta\left(n+c_{2}\right)\right] \cos \left[\beta\left(n+c_{2}\right)\right] \sum_{k=1}^{n / 2} B_{k}\left(\cos \left[\beta\left(n+c_{2}\right)\right]\right)^{2(k-1)} .
\end{aligned}
$$

Note that these four solutions are valid in case $\mu_{1}, \mu_{2}<0$. However, if $\mu_{1}$ and $\mu_{2}$ have opposite signs, say $\mu_{1}<0, \mu_{2}>0$, then the solution is given by

$$
\begin{aligned}
f_{n} & =\sum_{k=1}^{n / 2+1} \frac{A_{k}}{\left(\operatorname{dn}\left[\beta\left(n+c_{2}\right), m\right]\right)^{2(k-1)}}, \\
g_{n} & =\sqrt{m} \operatorname{sn}\left[\beta\left(n+c_{2}\right), m\right] \sum_{k=2}^{n / 2+1} \frac{B_{k}}{\left(\operatorname{dn}\left[\beta\left(n+c_{2}\right), m\right]\right)^{2(k-1)}} .
\end{aligned}
$$

For this solution, $f_{n}, g_{n}$ and hence $u_{n}, v_{n}$ satisfy the boundary condition (11). In the limit $m=1$, this solution goes over to the hyperbolic solution

$$
\begin{aligned}
f_{n} & =\sum_{k=1}^{n / 2+1} A_{k}\left(\cosh \left[\beta\left(n+c_{2}\right)\right]\right)^{2(k-1)}, \\
g_{n} & =\tanh \left[\beta\left(n+c_{2}\right)\right] \sum_{k=2}^{n / 2+1} B_{k}\left(\cosh \left[\beta\left(n+c_{2}\right)\right]\right)^{2(k-1)} .
\end{aligned}
$$

On substituting any of the expressions for $f_{n}, g_{n}$ as given by Eqs. (40) to (45) in Eq. (6) we again obtain $n+1$ equations which determine the $n+1$ parameters $A_{k}, B_{k}$. Some of these relations are

$$
\begin{aligned}
& A_{n / 2+1}^{2}=B_{n / 2}^{2} \\
& 2 A_{n / 2+1} A_{n / 2}+B_{n / 2}^{2}=2 B_{n / 2} B_{n / 2-1}, \\
& A_{n / 2}^{2}+2 A_{n / 2+1} A_{n / 2-1}+2 B_{n / 2} B_{n / 2-1}=B_{n / 2-1}^{2}+2 B_{n / 2} B_{n / 2-2}, \\
& B_{n / 2-1}^{2}+2 A_{n / 2+1} A_{n / 2-2}+2 B_{n / 2} B_{n / 2-2} \\
& +2 A_{n / 2} A_{n / 2-1}=2 B_{n / 2-1} B_{n / 2-2}+2 B_{n / 2} B_{n / 2-3}, \\
& A_{1}^{2}=1, \quad B_{1}^{2}+2 A_{1} A_{2}=0, \quad 2 A_{1} A_{3}+A_{2}^{2}+2 B_{1} B_{2}=B_{1}^{2}, \\
& B_{2}^{2}+2 A_{2} A_{3}+2 A_{1} A_{4}+2 B_{2} B_{2}=2 B_{1} B_{2} .
\end{aligned}
$$


On comparing these results with the exact expressions for $n=2,4$, we conjecture the following general results for arbitrary even $n$ :

$$
\begin{aligned}
& A_{n / 2+1}=B_{n / 2}=2^{n-1}, \quad A_{n / 2}=-n 2^{(n-3}, \quad B_{n / 2-1}=-(n-2) 2^{(n-3)}, \\
& A_{n / 2-1}=\frac{n(n-3)}{2 !} 2^{(n-5)}, \quad B_{n / 2-2}=\frac{(n-3)(n-4)}{2 !} 2^{(n-5)}, \\
& A_{n / 2-2}=-\frac{n(n-4)(n-5)}{3 !} 2^{(n-7)}, \quad B_{n / 2-3}=-\frac{(n-4)(n-5)(n-6)}{3 !} 2^{(n-7)}, \\
& \sum_{i=1}^{n / 2+1} A_{i}=1, \quad \sum_{i=1}^{n / 2} B_{i}=n .
\end{aligned}
$$

Further, depending on if $n=4 k+2$ or $4 k+4$ we have the following results $(k=0,1,2, \ldots)$ :

$$
\begin{gathered}
A_{1}=-1, \quad B_{1}=n, A_{2}=\frac{n^{2}}{2}, \quad \text { if } n=4 k+2, \\
A_{1}=+1, \quad B_{1}=-n, \quad A_{2}=-\frac{n^{2}}{2}, \quad \text { if } n=4 k+4 .
\end{gathered}
$$

In fact looking at these general results, we conjecture the following expressions for various $A_{i}, B_{i}$.

$$
\begin{gathered}
A_{n / 2-k+1}=(-1)^{k} \frac{n(n-k-1)(n-k-2) \ldots(n-2 k+1)}{k !} 2^{n-2 k-1}, \quad k=0,1,2, \ldots, n / 2, \\
B_{n / 2-k}=(-1)^{k} \frac{(n-k-1)(n-k-2) \ldots(n-2 k)}{k !} 2^{n-2 k-1}, \quad k=0,1,2, \ldots, n / 2-1 .
\end{gathered}
$$

It can be verified that for $n=2,4$, the $A_{i}, B_{i}$ which followfrom Eqs. (46) to (49) agree with the values obtained independently by us above as well as in[1]. Further, for low values of (even) $n$, it can be numerically checked that the $A_{i}, B_{i}$ which follow from Eqs. (50) and (51) indeed satisfy the various relations, which follow by demanding the validity of the constraint relation (6).

In the next section, we will prove that our conjectures hold for all $n$. But first, using Eqs. (49), (50), and (51), we predict that the following identities hold for all even $n$ :

$$
\begin{gathered}
\sum_{k=0}^{n / 2}(-1)^{k} \frac{n(n-k-1)(n-k-2) \ldots(n-2 k+1)}{k !} 2^{n-2 k-1}=1 . \\
\sum_{k=0}^{n / 2-1}(-1)^{k} \frac{(n-k-1)(n-k-2) \ldots(n-2 k)}{k !} 2^{n-2 k-1}=n .
\end{gathered}
$$

These identities will naturally lead to the proof of our conjectures.

As mentioned earlier, since the field Eqs. (4) and (5) are invariant under $f_{n} \rightarrow \pm f_{n}, g_{n} \rightarrow \pm g_{n}$, hence one can trivially write down three other solutions in both odd and even $n$ cases. 


\section{Connection to Chebyshev Polynomials}

In the previous section, we have defined two families $f_{n}, g_{n}$ and conjectured that they lead to the solutions of the constraint relation (6). Here, $f_{n}$ is a polynomial, and $g_{n}$ equals a polynomial times an extra factor, and we took their common argument to be a Jacobi elliptic function. We now prove that these proposed functions do indeed give rise to solutions for all $n$. To do so, we take a closer look at the identities stated in Eqs. (38), (39), (52), and (53). If we look at Eqs. (38) and (52) then we notice that in both the cases, the summation goes from $k=0$ to $k=m$, where $n=2 m+1$ in Eq. (38) and $n=2 m$ in Eq. (52). In other words, in both cases the summation goes from $k=0$ to $\lfloor n / 2\rfloor$. Thus Eqs. (38) and (52) change to the odd and even cases of

$$
\sum_{l=0}^{\lfloor n / 2\rfloor}\left(\begin{array}{c}
n-l \\
l
\end{array}\right) \frac{(-1)^{l} n 2^{n-2 l}}{2(n-l)}=1 .
$$

It turns out that replacing the 2 in the numerator by other bases yields other similar identities as well, that can be numerically verified for small $n$. To simplify the notation, define

$$
f_{n}(x):=\sum_{l=0}^{\lfloor n / 2\rfloor}\left(\begin{array}{c}
n-l \\
l
\end{array}\right) \frac{(-1)^{l} n(2 x)^{n-2 l}}{2(n-l)}
$$

where $x \in \mathbb{R}$. Our identities (38) and (52) say that $f_{n}(1)=1$. But before we prove this, we remark that we can also compute $f_{n}(x)$ for other values of $x$ and low values of $n$. We omit writing the details down, but doing so leads to the following (conjectured) identities:

$$
f_{n}(1)=1=\cos (2 n \pi), \quad f_{n}(1 / 2)=\cos (n \pi / 3), \quad f_{n}(0)=\cos (n \pi / 2),
$$

Similarly, it is possible to propose closed-form expressions for $f_{n}(-1 / 2)$ and $f_{n}(-1)$ as well. The connection between the terms on both sides in Eq. (56) is made by noting that

$$
1=\cos (2 \pi), \quad 1 / 2=\cos (\pi / 3), \quad 0=\cos (\pi / 2) .
$$

This leads to the following result for all $x$.

Theorem 1. For any $\theta \in[0, \pi]$,

$$
f_{n}(\cos (\theta))=\cos (n \theta)
$$

More generally, for any $x \in \mathbb{R}, f_{n}(x)=T_{n}(x)$, where $T_{n}$ is the $n$th Chebyshev polynomial of the first kind. 
Proof: We make use of an "explicit formula" on Chebyshev polynomials:

$$
T_{n}(x)=\sum_{l=0}^{\lfloor n / 2\rfloor} \frac{(-1)^{l} n}{2} \frac{(n-l-1) !}{l !(n-2 l) !}(2 x)^{n-2 l} .
$$

Simplifying the right-hand side, we obtain the desired result. Note that for $x \in[-1,1], x=\cos (\theta)$ for a unique $\theta \in[0, \pi]$, and then the definition of $T_{n}$ proves that $f_{n}(\cos (\theta))=\cos (n \theta)$.

It is also easy to compute that when $x=1,1 / 2$, or $0, T_{n}(x)=1, \cos (n \pi / 3)$, or $\cos (n \pi / 2)$ respectively, as claimed in Eq. (56).

Similarly, if we look at Eqs. (39) and (53), then we observe that both these cases change to the odd and even cases of

$$
\frac{1}{2} \sum_{l=0}^{\lfloor n / 2\rfloor}\left(\begin{array}{c}
n-l-1 \\
l
\end{array}\right)(-1)^{l} 2^{n-2 l}=n .
$$

It remains to compute the above series. More generally, we prove:

Theorem 2. Let

$$
g_{n}(x)=\frac{1}{2} \sum_{l=0}^{\lfloor n / 2\rfloor}\left(\begin{array}{c}
n-l-1 \\
l
\end{array}\right)(-1)^{l}(2 x)^{n-2 l} .
$$

Then for all $x \in \mathbb{R}, g_{n}(x)=x U_{n-1}(x)$, where $U_{n}(x)$ are the Chebyshev polynomials of the second kind.

Proof: We start from Eq. (59). On differentiating both sides, we have

$$
\begin{aligned}
T_{n}^{\prime}(x) & =\sum_{l=0}^{\lfloor n / 2\rfloor}(-1)^{l} \frac{n(n-l-1) !}{l !(n-2 l) !}(n-2 l)(2 x)^{n-2 l-1} \\
& =\frac{n}{x} g_{n}(x) .
\end{aligned}
$$

Simplifying, we get:

$$
g_{n}(x)=\frac{x}{n} \cdot T_{n}^{\prime}(x)=x U_{n-1}(x),
$$

where the second equality is standard.

It remains to prove the original identity. But the summation is simply $g_{n}(1)$, and it is well known that $U_{n}(1)=n+1$ for all $n$. Hence

$$
g_{n}(1)=U_{n-1}(1)=n .
$$


In fact it is now clear that for arbitrary $n, f_{n}$ and $g_{n}$ (as given by Eqs. (55) and (61) respectively, for any even or odd integer $n$ ) are simply

$$
f_{n}=T_{n}(y), \quad g_{n}=\left(1-y^{2}\right)^{1 / 2} U_{n-1}(y),
$$

where $y$ is one of the following:

$$
\begin{array}{lll}
\operatorname{dn}\left[\beta\left(n+c_{2}\right), m\right], & \operatorname{cn}\left[\beta\left(n+c_{2}\right), m\right], \quad \operatorname{sech}\left[\beta\left(n+c_{2}\right)\right], & \cos \left[\beta\left(n+c_{2}\right)\right], \\
\frac{1}{\operatorname{dn}\left[\beta\left(n+c_{2}\right), m\right]}, & \cosh \left[\beta\left(n+c_{2}\right)\right] .
\end{array}
$$

Note that

$$
f_{n}^{2}(y)+g_{n}^{2}(y)=T_{n}^{2}(y)+\left(1-y^{2}\right) U_{n-1}^{2}(y)=1 .
$$

In other words, the solutions of the coupled equations are simply Chebyshev polynomials with argument in terms of Jacobi elliptic functions. Actually, once one realizes this, then the structure of other solutions is also simplified. In particular, it is known that

$$
T_{n}(\cos (\theta))=\cos (n \theta), \quad T_{n}(\cosh (x))=\cosh (n x) .
$$

Thus, $f_{n}(x)=\cos (n \theta)$ or $\cosh (n x)$, which makes $g_{n}(x)=\sin (n \theta)$ or $\sinh (n x)$ respectively. Thus, one now has a better understanding of the solutions (31), (45), (29) and (43).

\section{$5 \quad$ Other Coupled Models}

We now consider the coupled Ablowitz-Ladik (AL), coupled $\phi^{6}$ and coupled $\phi^{4}$ models as discussed in our previous publication [1] and show that all these models also admit solutions in terms of Lamé polynomials of arbitrary order.

\subsection{Solutions of a Coupled AL Model}

As shown in our previous publication [1, in the special case when $\nu_{1}=2 \mu_{1}$ and $\nu_{2}=2 \mu_{2}$, the coupled Salerno model as given by Eqs. (12) and (2) reduces to the coupled AL model with the field equations

$$
i d u_{n} / d t+\left[u_{n+1}+u_{n-1}-2 u_{n}\right]+\left(\mu_{1}\left|u_{n}\right|^{2}+\mu_{2}\left|v_{n}\right|^{2}\right)\left[u_{n+1}+u_{n-1}\right]=0,
$$




$$
i d v_{n} / d t+\left[v_{n+1}+v_{n-1}-\frac{2 \mu_{2}}{\mu_{1}} v_{n}\right]+\left(\mu_{1}\left|u_{n}\right|^{2}+\mu_{2}\left|v_{n}\right|^{2}\right)\left[v_{n+1}+v_{n-1}\right]=0 .
$$

It is then clear that all the solutions of coupled Salerno model in terms of Lamé polynomials of arbitrary order, are automatically the solutions of the coupled AL model and further in this case, $\omega_{1}=2, \omega_{2}=\frac{2 \mu_{1}}{\mu_{2}}$.

\subsection{Solutions of a Coupled Discrete $\phi^{6}$ Model}

The field equations of the coupled discrete $\phi^{6}$ model discussed in our recent paper [1] are

$$
\begin{gathered}
\frac{1}{h^{2}}\left(\phi_{n+1}+\phi_{n-1}-2 \phi_{n}\right)=a_{1} \phi_{n}-b_{1} \phi_{n}^{3}+d \psi_{n}^{2} \phi_{n}+\left[c_{1} \phi_{n}^{4}+e \phi_{n}^{2} \psi_{n}^{2}+f \psi_{n}^{4}\right]\left[\phi_{n+1}+\phi_{n-1}\right], \\
\frac{1}{h^{2}}\left(\psi_{n+1}+\psi_{n-1}-2 \psi_{n}\right)=a_{2} \psi_{n}-b_{2} \psi_{n}^{3}+d \phi_{n}^{2} \psi_{n}+\left[c_{2} \psi_{n}^{4}+\frac{e}{2} \phi_{n}^{4}+2 f \phi_{n}^{2} \psi_{n}^{2}\right]\left[\psi_{n+1}+\psi_{n-1}\right] .
\end{gathered}
$$

As shown in [1], solutions to these coupled equations in terms of Lamé polynomials of order one and two are obtained in case

$$
\phi_{n}^{2}+\psi_{n}^{2}=\sqrt{\frac{1}{c_{1} h^{2}}}
$$

and further if

$$
c_{1}=c_{2}=f=\frac{e}{2}, \quad b_{1}=b_{2}=-d, \quad a_{1}=a_{2}, \quad c_{1} h^{2} b^{2}=1, \quad a_{1}+\frac{2}{h^{2}}=\frac{b_{1}}{\sqrt{h^{2} c_{1}}} .
$$

It is then clear that the solutions (26) to (28) and (40) to (42) in terms of Lamé polynomials of arbitrary order obtained in the case of the coupled Salerno model will also be the solutions of this coupled model.

\subsection{Solutions for a Coupled Discrete $\phi^{4}$ Model}

In our recent publication [1] we considered the following coupled $\phi^{4}$ model

$$
\begin{aligned}
& \frac{1}{h^{2}}\left(\phi_{n+1}+\phi_{n-1}-2 \phi_{n}\right)-2 \alpha_{1} \phi_{n}-\left[2 \beta_{1} \phi_{n}^{2}+\gamma \psi_{n}^{2}\right]\left[\phi_{n+1}+\phi_{n-1}\right]=0, \\
& \frac{1}{h^{2}}\left(\psi_{n+1}+\psi_{n-1}-2 \psi_{n}\right)-2 \alpha_{2} \psi_{n}-\left[2 \beta_{2} \psi_{n}^{2}+\gamma \phi_{n}^{2}\right]\left[\psi_{n+1}+\psi_{n-1}\right]=0,
\end{aligned}
$$

and showed that solutions to these coupled equations can be obtained in terms of Lamé polynomials of order one and two provided

$$
\phi_{n}^{2}+\psi_{n}^{2}=\frac{1}{2 \beta_{1} h^{2}}
$$


and further if

$$
2 \beta_{1}=2 \beta_{2}=\gamma, \quad \alpha_{1}=\alpha_{2}=-\frac{1}{h^{2}} .
$$

It is then clear that the solutions (26) to (29) and (40) to (43) in terms of Lamé polynomials (or corresponding hyperbolic and trigonometric polynomials) of arbitrary order obtained in the case of the coupled Salerno model will also be the solutions of this coupled model.

\section{Summary}

In this paper we have shown that for a number of coupled discrete models, e.g., coupled Salerno, coupled Ablowitz-Ladik, coupled $\phi^{6}$, coupled $\phi^{4}$, there are solutions in terms of Lamé polynomials of arbitrary order while the uncoupled equations do not admit solutions in terms of Lamé polynomials of order two and higher. In particular, we showed that the Lamé polynomials can be reexpressed as Chebyshev polynomials of the relevant Jacobi elliptic function. Many of these solutions are relevant to physical contexts ranging from ferroelectric materials [8, 9, 10] to multiferroics [5, 6, 7] to a variety of models in field theory [11, 15] in addition to various discrete contexts [12, 13, 14].

It is important to emphasize that the stability of the various solutions found here remains an open issue to be explored numerically, particularly since the soliton solutions obtained above are of arbitrary width. Besides, a comparative study of the scattering of solitons of different discrete models is an important issue with these static solutions boosted with a certain velocity. Similarly, the Peierls-Nabarro (discreteness) barrier for the solutions remains to be explored. However, since all our solutions have discrete translational invariance (i.e., they are valid for arbitrary $c_{2}$ ), it is likely that for all our solutions the Peierls-Nabarro barrier may be zero. However, this issue needs to be explored carefully and we intend to do so in the near future.

\section{Acknowledgment}

This work was supported in part by the U.S. Department of Energy. 


\section{References}

[1] A. Khare and A. Saxena, Pramana (J. Phys.) 77, (2011).

[2] A. Khare and A. Saxena, J. Phys. A42, 335401 (2009).

[3] A. Khare, K. Ø. Rasmussen, M. Salerno, M. R. Samuelsen, and A. Saxena, Phys. Rev. E 74, 016607 (2006).

[4] H. Schmid, Ferroelectrics 162, 317 (1994); N. A. Spaldin and M. Fiebig, Science 309, 391 (2005).

[5] T. Kimura, T. Goto, H. Shintani, K. Ishizaka, T. Arima, and Y. Tokura, Nature 426, 55 (2003).

[6] M. Fiebig, Th. Lottermoser, and R. V. Pisarev, J. Appl. Phys. 93, 8194 (2003).

[7] S. H. Curnoe and I. Munawar, Physica B 378-380, 554 (2006).

[8] S. Aubry and R. Pick, Ferroelectrics 8, 471 (1973).

[9] T. Abel and R. Siems, Ferroelectrics 153, 177 (1994).

[10] A. A. Kornyshev, D. A. Kossakowski, and S. Leikin, J. Chem. Phys. 97, 6809 (1992).

[11] A. N. Das and B. Ghosh, J. Phys. C 16, 1803 (1983).

[12] J. Cuevas, Q. E. Hoq, H. Susanta, and P. G. Kevrekidis, Physica D 238, 2216 (2009).

[13] V. M. Rothos and P. G. Kevrekidis, J. Phys. A 40, 4553 (2007).

[14] P. G. Kevrekidis, The Discrete Nonlinear Schrödinger Equation, (Springer-Verlag, Heidelberg, 2009).

[15] R. Rajaraman, Phys. Rev. Lett. 42, 200 (1979).

[16] C. Matsuoka and K. Nozaki, Phys. Lett. A 185, 310 (1994).

[17] C. S. Lai, Canad. J. Phys. 58, 443 (1980).

[18] N. N. Rao, J. Phys. A 22, 4813 (1989). 
[19] X. Y. Wang, B. C. Xu, and P. L. Taylor, Phys. Lett. A 173, 30 (1993).

[20] X. W. Huang, J. H. Han, K. Y. Qian, and W. Qian, Phys. Lett. A 182, 300 (1993).

[21] D. Bazeia, M. J. Dos Santos, and R. F. Ribeiro, Phys. Lett. A 208, 84 (1995).

[22] Y. J. Zhu and S. Y. Lou, Commun. Theor. Phys. 30, 147 (1998).

[23] S. Y. Lou, J. Phys. A 32, 4521 (1999).

[24] D. B. Cao, Phys. Lett. A 296, 27 (2002).

[25] D. S. Li and H. Q. Zhang, Acta Phys. Sinica 52, 2373 (2003); ibid. 2379 (2003).

[26] C. P. Liu, Chaos Solit. Fract. 20, 619 (2004); Commun. Theor. Phys. 43, 13 (2005).

[27] S. K. Liu, Z. T. Fu, S. D. Liu, and Z. G. Wang, Phys. Lett. A 323, 415 (2004).

[28] A. Erdélyi, Higher Transcendental Functions (Bateman Project) vol. 3 (New York, McGraw-Hill, $1955)$.

[29] A. Khare and A. Saxena, J. Math. Phys. 49, 063301 (2008); arXiv:nlin.SI/0609013.

[30] A. Khare and A. Saxena, J. Math. Phys. 47, 092902 (2006).

[31] M. Salerno, Phys. Rev. A 46, 6856 (1992).

[32] M.J. Ablowitz and J.F. Ladik, J. Math. Phys. 17, 1011 (1977).

[33] R. Scharf and A.R. Bishop, Phys. Rev. A 43, 6535 (1991).

[34] B. A. Malomed and J. Yang, Phys. Lett. A 302, 163 (2002).

[35] A. Khare and U. Sukhatme, J. Math. Phys. 43, 3798 (2002); A. Khare, A. Lakshminarayan and U. Sukhatme, ibid 44, 1822 (2003); Pramana (J. Phys.) 62, 1201 (2004).

[36] A. Khare, K.O. Rasmussen, M.R. Samuelsen and A. Saxena, J. Phys. A 38, 807 (2005); ibid. A 42, $085002(2009)$. 